(2) Open Access Full Text Article

\title{
Brentuximab vedotin in Hodgkin lymphoma and anaplastic large-cell lymphoma: an evidence-based review
}

This article was published in the following Dove Press journal:

OncoTargets and Therapy

\section{Eva M Donato'}

Miguel Fernández-Zarzoso'

Jose Antonio Hueso'

Javier de la Rubia ${ }^{1,2}$

'Hematology Service, University Hospital Doctor Peset, Valencia, Spain; ${ }^{2}$ Department of Internal Medicine, Universidad Católica de Valencia "San Vicente Mártir," Valencia, Spain
Correspondence: Eva M Donato Hematology Service, University Hospital Doctor Peset. Av. de Gaspar Aguilar 90. 46017 Valencia, Spain Email eva_donato@yahoo.es

\begin{abstract}
Hodgkin lymphoma (HL) and anaplastic large-cell lymphoma (ALCL) account for $\sim 10 \%$ and $2 \%-3 \%$ of all cases of lymphoid neoplasms, respectively. Up to $30 \%$ of patients with HL are refractory or relapse after first-line therapy, and elderly patients with HL represent a subgroup of patients with suboptimal responses to the currently available treatments. Five-year overall survival for ALCL patients is $50 \%-80 \%$ with conventional chemotherapy. Therefore, new therapeutic approaches are needed for these groups of patients. Brentuximab vedotin is a chimeric IgG1 anti-CD30 antibody-drug conjugate that has all the features that are necessary to make a substantive difference with the standard therapies in patients with HL and ALCL: a novel mechanism of action, single-agent activity, non-cross-resistance, and safety both in the relapsed-refractory and in the front-line setting. This review provides an update of the results of the most relevant clinical trials including brentuximab vedotin for patients with HL and ALCL conducted to date.
\end{abstract}

Keywords: anaplastic large-cell lymphoma, anti-CD30 antibody, brentuximab vedotin, Hodgkin lymphoma

\section{Introduction}

Hodgkin lymphoma (HL) most commonly affects young adults and accounts for $\sim 10 \%$ of all cases of lymphoma in the United States and represents $5 \%$ of all annual deaths from lymphoma. ${ }^{1}$ Cutaneous and systemic anaplastic large-cell lymphomas (ALCL) are a subtype of peripheral T-cell lymphomas that account for $2 \%-8 \%$ of all lymphoid neoplasms. ${ }^{2,3}$ Primary systemic ALCL represents $2 \%-8 \%$ of non-Hodgkin lymphomas in adults and $20 \%-30 \%$ of large-cell lymphomas in children. ${ }^{3}$ ALK expression can be observed in up $85 \%$ of ALCL while the remaining cases are considered ALK-negative. Both subtypes show different features and are considered as 2 different entities in the updated World Health Organization classification. ${ }^{3}$

Currently available front-line therapies can cure most of the younger patients with HL, but up to $30 \%$ of them are refractory or relapse after first-line therapy. The standard of care for these patients is second-line chemotherapy followed by autologous stem cell transplantation (ASCT), and it is associated with a 50\%-55\% cure rate, but new treatments are need for relapsed and refractory HL (RRHL) patients after ASCT. Though HL predominantly affects young people, elderly patients represent a subgroup of patients who are the most challenging to treat and there are scarce options for those who fail first-line therapy. Therefore, improved outcomes with the introduction of agents with new mechanism are also clearly needed for these patients. Finally, 5-year 
overall survival (OS) for ALK-positive patients is 70\%-80\% with conventional chemotherapy but it is only $50 \%$ for ALKnegative patients. ${ }^{4}$ Few effective treatments are available for relapsed or refractory ALK-negative patients, and novel therapeutic options are warranted to improve prognosis in this uncommon subtype of lymphoma.

CD30 is a $120-k d$ transmembrane cytokine receptor of the tumor necrosis factor receptor family ${ }^{4}$ that is expressed in the lymphoid cells of most patients with HL and ALCL. CD30 expression is confined to activated lymphocytes and eosinophils, usually on lymphoid tissues, but not on peripheral blood cells. CD30 has thus been identified as an attractive target for therapy. Brentuximab vedotin (BV) is a chimeric IgG1 anti-CD30 antibody-drug conjugated by a protease-cleavable linker to the microtubule-disrupting agent monomethyl auristatin $\mathrm{E}$ that has shown impressive antitumor activity in both HL and ALCL. ${ }^{5}$ Targeted delivery of monomethyl auristatin E to CD30 expressing tumor cells is the primary mechanism of action of BV. Additional mechanisms of tumor cell death that may contribute to the clinical activity of this drug include antibody-dependent cellular phagocytosis, immunogenic cell death, and the bystander effect. Availability of BV has become an important step forward in the improvement of the outcome of patients with relapsed and refractory HL. ${ }^{6}$ Furthermore, the significant clinical activity observed and the good tolerability of BV has allowed for widespread investigation and use of BV among a variety of lymphoma patients, and several groups are testing BV-based therapies in the management of newly diagnosed patients with HL and ALCL, with encouraging preliminary results.

In this review, the use of BV-based therapies for the treatment of relapsed/refractory HL and ALCL and the most common BV-associated side effects will be reviewed, with the addition of some perspectives of how this new drug would impact the clinical management of these patients in the future.

\section{Clinical efficacy of BV in HL Relapsed/refractory HL}

\section{Approved indications}

In the pre-BV era, patients with RRHL after ASCT had few therapeutic options with median OS ranging from 10.5 to 27.6 months. ${ }^{7,8}$ This scenario, however, changed with the introduction of $\mathrm{BV}$.

In a Phase II, single-arm study, testing the efficacy and safety of BV monotherapy, 102 patients with RRHL after ASCT were included. BV was administered at a dose of $1.8 \mathrm{mg} / \mathrm{kg}$, once every 3 weeks, for up to 16 cycles. ${ }^{9}$ Overall response rate (ORR) was $75 \%$, with a $34 \%$ complete response (CR) rate. Notably, $71 \%$ of patients in the trial had refractory disease at the time of transplant, highlighting the remarkable activity of single-agent BV in patients with advanced-stage disease who are refractory to current standard options, including high-dose therapy. This trial was the first to demonstrate substantial activity of BV in patients with advanced disease and limited treatment options and led to the accelerated approval of BV by the US Food and Drug Administration (2011) and the European Medicines Agency (2012) for the treatment of patients with HL who have relapsed after an ASCT or following 2 prior lines of therapy.

The long-term follow-up results of the pivotal trial showed a 5-year progression-free survival (PFS) of $22 \%$ and an OS of $41 \%$. Patients who achieved CR $(n=34)$ had PFS and OS rates of 52\% (95\% confidence interval [CI], 34-69) and $64 \%$ (95\% CI, 48-80), respectively. ${ }^{10}$ In this trial, most patients in CR (28 out of the 34 patients) did not receive consolidation with allogeneic transplantation and the PFS and OS rates in these patients were $48 \%$ and $60 \%$, respectively. Several reports have also addressed the efficacy of singleagent BV in RRHL patients treated outside conventional clinical trials. Overall, these studies show clinical outcomes similar to those reported by the pivotal trial, with $\mathrm{CR}$ rates ranging from $18 \%$ to $46 \%$ and with PFS ranging from 5.7 to 10.2 months. $^{5}$

The Phase III randomized, double-blind, placebo-controlled AETHERA trial studied the role of consolidation treatment with BV to prevent relapse after ASCT. ${ }^{11}$ Patients included in the trial had at least 1 of the following risk factors: primary refractory HL, relapsed HL (initial remission duration of $<12$ months), or extranodal involvement at relapse. Patients were randomized to receive either BV $(\mathrm{n}=165)$ at a dose of $1.8 \mathrm{mg} / \mathrm{kg}$ on day 1 of each 21 -day cycle for up to 16 cycles or placebo $(n=165)$, starting 30-45 days after ASCT. The median PFS was 42.9 and 24.1 months for those patients receiving $\mathrm{BV}$ and placebo, respectively $(\mathrm{HR}=0.57, P=0.0013)$ with a 3 -year PFS rate of $61 \%(95 \%$ CI, 52-68) for the BV group and 43\% (95\% CI, 36-51) for the placebo arm. ${ }^{12}$ There was significantly more neuropathy (56\%) and neutropenia (35\%) in the BV arm. No differences in OS were observed between both groups of patients, probably because most patients in the placebo arm received BV as rescue therapy at relapse. Pretransplant PET was not required in the trial, and therefore the potential benefit of the role of post-transplant maintenance with BV in patients with PETconfirmed CR remains unknown. However, the analysis of the subgroup of patients with PET-negative pre-ASCT did 
not show differences in terms of PFS when compared with patients receiving placebo.

\section{BV-based combinations}

It is generally accepted that in patients with $\mathrm{HL}$, remission status pre-ASCT is highly predictive of outcome, and the incorporation of $\mathrm{BV}$ into a rescue therapy regimen before transplant could provide a unique opportunity to improve response. The current approved indications of BV do not include transplant-eligible patients refractory to second-line therapy, and several groups have tested the usefulness of BV either alone or in combination as pretransplant rescue therapy for this group of patients.

The role of second-line therapy before ASCT with BV monotherapy was analyzed by Chen et al in a series of 37 patients with RRHL. In this trial, patients received 4 doses of standard BV. ${ }^{13}$ The ORR was $68 \%$ and 18 patients ( 13 in CR) proceeded to ASCT without further combination chemotherapy. This trial showed that monotherapy with BV can be effective and safely administered in RRHL patients as a bridge to ASCT. Additional studies have reported results of the administration of BV either sequentially or in combination with several chemotherapeutic regimens as part of a salvage strategy prior to ASCT including gemcitabine-based, ${ }^{14}$ ifosfamide, carboplatin, and etoposide (ICE), ${ }^{15,16}$ bendamustine,,${ }^{17}$ or etoposide, methylprednisolone, cytarabine, and cisplatin (ESHAP) therapies, ${ }^{18}$ achieving PET-negativity prior to ASCT ranging from $70 \%$ to $76 \%{ }^{14-18}$ (Table 1). Interestingly, and although studies cannot be directly compared, results show that patients in CR after singleagent BV or after BV-based combinations achieve similar results post-ASCT with PFS higher than $80 \%$ at 2 years.

Finally, early data indicate that the combination of BV with the immune checkpoint inhibitor nivolumab is an active and well-tolerated salvage combination in the RRHL setting with an ORR of $100 \%$ and $\mathrm{CR}$ rates ranging from 50 to $62.5 \% .^{19,20}$ A Phase III confirmatory study comparing nivolumab plus BV to BV alone for patients with RRHL following ASCT or who are transplant ineligible (CheckMate 812, NCT03138499) is currently ongoing. In addition, a randomized Phase III trial comparing pembrolizumab versus BV for RRHL patients after ASCT or those who are ASCT ineligible is also currently active (NCT02684292).

\section{Retreatment with BV}

A Phase II study investigated the safety and antitumor activity of retreatment with BV in patients who previously achieved CR or partial response (PR) with this drug. ${ }^{21}$ The reported ORR was $60 \%(30 \% \mathrm{CR})$ with a median duration of response for patients achieving PR or better of 9.2 months.

\section{$B V$ in the front-line setting}

\section{Elderly patients}

Given the response rates observed with BV in RRHL patients and the high toxicity observed with standard front-line adriamycin, bleomycin, vinblastine, and dacarbazine (ABVD) chemotherapy in elderly patients, a reasonable option could be to test single-agent BV in elderly patients with newly diagnosed HL. The efficacy and safety of BV as front-line therapy was reported in a Phase II study including $27 \mathrm{HL}$ patients over the age of 60 years. ${ }^{22}$ The ORR and CR were $92 \%$ and $73 \%$, respectively. The median PFS was 10.5 months (range, 2.6-22.3) for all efficacy-evaluable patients $(n=26)$ and 11.8 months (range, 4.1-22.3) for those achieving CR. Similar results were confirmed by another study including 38 patients who were considered unfit for standard chemotherapy. The complete metabolic response rate following cycle 4 was

Table I Results of the studies including BV sequentially or in combination with chemotherapy as part of a salvage strategy prior to $\mathrm{SCT}^{\mathrm{a}}$

\begin{tabular}{|c|c|c|c|c|c|c|}
\hline Author & $\begin{array}{l}\text { Number } \\
\text { of patients }\end{array}$ & $\begin{array}{l}\text { Objective } \\
\text { response (\%) }\end{array}$ & $\begin{array}{l}\text { PET-negative } \\
\text { prior to SCT (\%) }\end{array}$ & $\begin{array}{l}\text { SCT } \\
\text { performed (\%) }\end{array}$ & PFS & OS \\
\hline \multirow[t]{2}{*}{ Michallet et al ${ }^{15}$} & 11 & CR: 73 & 73 & 100 & II.2 months & I year: $60 \%$ \\
\hline & & PR: 27 & & & $(95 \% \mathrm{Cl}, \mathrm{NA})$ & $(95 \% \mathrm{Cl}, \mathrm{NA})$ \\
\hline \multirow[t]{2}{*}{ Moskowitz et a $\left.\right|^{16}$} & 45 & CR: 76 & 76 & 98 & 2 years: $80 \%$ & 2 years: $95 \%$ \\
\hline & & PR: 22 & & & (95\% Cl, 68-92) & $(95 \% \mathrm{Cl}, 88-100)$ \\
\hline \multirow[t]{2}{*}{ Cassaday et $\mathrm{al}^{17}$} & 16 & CR: 88 & NA & 75 & NA & NA \\
\hline & & PR: 6 & & & & \\
\hline \multirow[t]{2}{*}{ La Casce et $\mathrm{al}^{18}$} & 55 & CR: 74 & 74 & 73 & I year: $80 \%$ & NA \\
\hline & & PR: 19 & & & $(95 \% \mathrm{Cl}, 60-90)$ & \\
\hline \multirow[t]{2}{*}{ Garcia-Sanz et al ${ }^{19}$} & 66 & CR: 70 & 70 & 92 & year: $87 \%^{\mathrm{a}}$ & I year: $90 \%$ b \\
\hline & & PR: 26 & & & $(95 \% \mathrm{Cl}, \mathrm{NA})$ & $(95 \% \mathrm{Cl}, \mathrm{NA})$ \\
\hline
\end{tabular}

Notes: aAdapted from Donato EM, Fernández-Zarzoso M, De La Rubia J. Immunotherapy for the treatment of Hodgkin lymphoma. Expert Rev Hematol. 20I7; I0(5):4I7-423, with permission from Taylor \& Francis Ltd., http://www.informaworld.com. ${ }^{46}$ bData available in 47 patients.

Abbreviations: BV, brentuximab vedotin; SCT, stem cell transplantation; PFS, progression-free survival; OS, overall survival; Cl, confidence interval; NA, not available; CR, complete response; PR, partial response. 
$26 \%$, with a median PFS was 7 months. Finally, 28 of the 31 evaluable patients had progressed at the time of the report. ${ }^{23}$

To improve these results, combinations of BV plus bendamustine or dacarbazine have been tested as front-line induction therapy in elderly patients with HL. ${ }^{24,25}$ The association of BV plus dacarbazine showed an ORR of $100 \%$ and a CR rate of $62 \%$. With a median follow-up time of 21.6 months, median PFS was 17.9 months (not reached for patients in CR and 10.8 months for patients in PR). BV plus bendamustine has been associated with an ORR of $100 \%$ and a $\mathrm{CR}$ rate of $88 \%$ but with a much higher incidence of serious adverse events (AEs; 65\% of patients). According to the available results, BV plus dacarbazine seems to be a better tolerated regimen and a more suitable front-line therapy for this subgroup of patients. Finally, the combination of BV and nivolumab is also being explored as first-line therapy in older patients in a Phase II study (NCT02758717), but no data are available yet.

\section{Front-line treatment with BV}

These results of BV in the relapsed and refractory setting prompted the launch of several trials focused on the role of BV as front-line therapy for patients with HL. A Phase I study evaluated the association of BV with ABVD in 25 patients with advanced-stage HL. Preliminary results showed an incidence of pulmonary toxicity of $44 \%$ including 2 deaths. ${ }^{26}$ A second cohort tested the combination of adriamycin, vinblastine, and dacarbazine (AVD) plus BV in an additional 26 patients showing that BV can be safely combined with AVD at full therapeutic doses with a CR rate of $96 \%$ and an estimated 5-year PFS and OS of $92 \%$ and 100\%, respectively. ${ }^{27}$

BV plus AVD combination has also been tested in 29 patients with early-stage classical HL with unfavorable risk features. ${ }^{28}$ Patients received 4 cycles of BV and AVD, and 25 of them also underwent site radiation therapy (30 Gy) after induction, with every patient achieving CR. With a median follow-up of 18.8 months, 1-year PFS was 93.3\% (95\% CI, 84-102). The role of the combination of AVD plus BV versus ABVD in patients with newly diagnosed stage III or IV classic HL was examined in the large international prospective randomized ECHELON-1 trial. ${ }^{29}$ In this study, 664 patients received BV plus AVD and 670 received ABVD. At a median follow-up of 24.9 months, 2-year PFS in the BV plus AVD and ABVD groups were $82.1 \%$ (95\% CI, 78.7-85) and 77.2\% (95\% CI, 73.7-80.4), respectively. BV plus AVD showed superior efficacy to $A B V D$ in these patients, with a 4.9 percentage-point lower combined risk of progression, death, or non-CR, and use of subsequent anticancer therapy at 2 years.
In another Phase II trial, 104 newly diagnosed patients with HL were randomly allocated to receive 6 cycles of BV, etoposide, cyclophosphamide, doxorubicin, procarbazine, and prednisone (BrECAPP) or BV, etoposide, cyclophosphamide, doxorubicin, dacarbazine, and dexamethasone (BrECADD). ${ }^{30}$ Results showed that both combinations produced a similar CR ( $86 \%$ for BrECAPP and 88\% for BrECADD) and 1-year PFS (98\% for BrECAPP and 94\% for BrECADD) rates than conventional bleomycin, etoposide, adriamycin, cyclophosphamide, vincristine, procarbazine, and prednisone (BEACOPP). A randomized, Phase III trial is currently comparing the efficacy and safety of first-line BrECADD and BEACOPP in advanced-stage HL (HD21 trial, NCT02661503).

\section{Clinical efficacy of BV in systemic ALCL}

CD30 is expressed on the surface of cells in ALCL, a subtype of peripheral T-cell lymphomas, representing $\sim 2 \%-3 \%$ of all lymphoid neoplasms. ${ }^{2}$ Cases of ALK-positive ALCL are associated with a characteristic translocation, $\mathrm{t}(2 ; 5)$ (p23;q35), resulting in a fusion gene, NPM-ALK, encoding a protein with tyrosine kinase activity. CD30 and ALK are key molecules involved in the pathogenesis, diagnosis, and treatment of ALCL, and it has been clearly established that ALK-positive patients have fared better than ALK-negative patients due to their high sensitivity to anthracycline-based chemotherapy. ${ }^{31}$ Given this favorable outcome, cyclophosphamide, vincristine, doxorubicin, and prednisone (CHOP) is considered the standard front-line therapy for ALKpositive ALCL.

\section{Relapsed and refractory ALCL}

About half of patients with systemic ALCL will relapse after front-line treatment. Most adults with relapsed and refractory ALCL (RRALCL) have poor outcomes and consolidation with ASCT seems to be the standard therapy. ${ }^{32-34}$ However, given the strong and uniform expression of CD30, the antibody-drug conjugate BV has been widely tested in the relapse and refractory setting. Initially, 45 patients with relapsed CD30-positive HL and systemic ALCL were included in a Phase I multicenter, dose-escalation study of BV monotherapy. ${ }^{35}$ Two out of the 45 patients with systemic ALCL achieved CR, 1 in the $1.2 \mathrm{mg}$ cohort and 1 in the $2.7 \mathrm{mg}$ cohort (the maximum tolerated dose was $1.8 \mathrm{mg} / \mathrm{kg}$ given every 3 weeks). Another multinational, open-label, Phase II study evaluated the safety and efficacy of BV as single agent in 58 patients with RRALCL. ${ }^{36}$ The median age 
was 52 years (range, 14-76), and the median (range) of prior chemotherapy regimens was $2(1-6)$. Half of the patients were in relapse at study entry and $50 \%$ were refractory to their most recent therapy. Most patients (72\%) had ALK-negative ALCL. BV was administered at a dose of $1.8 \mathrm{mg} / \mathrm{kg}$, once every 3 weeks for up to 16 cycles, and the ORR was $86 \%$. Based on these findings, BV was approved by the US Food and Drug Administration in 2011 for the treatment of patients with RRALCL. The updated results of this clinical trial have been recently reported. ${ }^{37}$ With a 5-year follow-up, 38 (66\%) patients achieved $\mathrm{CR}$, and these objective responses were independent of ALK status or number of prior therapies. The estimated OS rate for the whole series was $79 \%$ and $25 \%$ for the subgroup of patients not achieving CR. In addition, PFS rate among CR patients was substantially higher than in total enrolled patients (57\% versus 39\%, respectively). BV therapy showed long-lasting CR both in the group of patients undergoing consolidation with ASCT and in those patients not receiving ASCT. Overall, 25 patients (43\%) had died, 14 of them due to disease progression.

Similar results have been reported by an Italian observational, multicenter, retrospective study including 40 patients with systemic ALCL. All these patients received treatment with single-agent $\mathrm{BV}$ at the time of relapse with an ORR of $62.5 \%$ (45\% CR and $17.5 \% \mathrm{PR}) .^{38}$

\section{Front-line BV in ALCL}

As in aggressive B-cell lymphoma, $\mathrm{CHOP}$ is considered the standard front-line therapy for patients with peripheral T-cell lymphomas. ${ }^{39,40}$ However, relapses are commonly observed, particularly in ALK-negative patients. To improve clinical results, front-line treatment combining BV with cyclophosphamide, doxorubicin, and prednisone (CHP) was evaluated in a Phase I clinical trial including 26 patients with CD30-positive peripheral T-cell lymphoma (19 with systemic ALCL). ${ }^{41}$ Patients received standard dose of BV with CHP on day 1 of each 21-day cycle. After 6 cycles, responding patients could receive up to 10 additional cycles of $\mathrm{BV}$ alone. With a median follow-up of 59.6 months, the objective response was $100 \%$ including $24 \mathrm{CR}$ and $2 \mathrm{PR}$ patients. The median PFS was 34.6 months and the estimated 5-year PFS was 47\% for patients with ALCL. The median OS was not reached, and the estimated 5-year OS was 79\%. Nine out of the 19 ALCL patients ( $3 \mathrm{ALK}+, 6 \mathrm{ALK}-$ ) remained in remission after 5 years of follow-up. Given those promising results, a Phase III, randomized clinical trial comparing front-line BV $+\mathrm{CHP}$ to $\mathrm{CHOP}$ for peripheral T-cell lymphomas is currently ongoing (ECHELON-2 trial, NCT01777152).

\section{Safety and BV-associated side effects}

In the Phase II pivotal study previously done in patients with RRHL, the most common grade 3 or grade 4 AEs observed were peripheral neuropathy $(\mathrm{PN})(8 \%)$, fatigue $(2 \%)$, neutropenia $(20 \%)$, and diarrhea $(1 \%) .{ }^{9}$ The 56 patients who experienced PN symptoms were followed for improvement and/or resolution. After 3 years of follow-up, PN resolved completely in $41(73 \%)$ patients and improved in the remaining $8(14 \%) .{ }^{10}$ Similar results have been described with BV in patients with relapsed (41\% of PN of any grade) $)^{37}$ and newly diagnosed systemic ALCL. ${ }^{41}$ Larger experiences with BV support PN and neutropenia as the most common adverse effects associated with this therapy. ${ }^{42,43}$ Like in younger patients, in elderly patients, PN (78\%), fatigue (44\%), and nausea (44\%) are the most commonly observed AEs with a low incidence of grade 2 or 3 treatment-related anemia and neutropenia ( 2 patients each). ${ }^{22}$ Finally, in the retreatment setting, single-agent BV was associated to a toxicity profile similar in type and frequency to that described in the pivotal trials but with a higher incidence of PN (28\% versus $11 \%$ for patients in the pivotal trial), mostly grade 1 or 2 in severity. ${ }^{21}$

Finally, preliminary results showed that the association of BV with bleomycin included in the ABVD regimen resulted in significant incidence of pulmonary side effects with up to $44 \%$ of patients developing some grade of lung toxicity including 2 deaths. ${ }^{26}$

\section{Quality of life in patients treated with BV}

Lymphoma patients have a high symptom burden which is associated with reduced overall health-related quality of life (HRQoL). Therefore, treatment objectives should be aimed to delay disease progression, optimize quality of response, and prolong OS coupled with maintaining HRQoL and minimizing treatment-related toxicity and discomfort.

In the 2 pivotal Phase II multicenter, open-label trials that evaluated the safety and efficacy of BV in patients with RRHL or systemic ALCL, ${ }^{9,36}$ results of HRQoL were evaluated in patients with long-term follow-up (median, 5 years) achieving overall objective response. ${ }^{44}$ Data were collected using a specifically designed, brief, 8-question survey that included patient-reported outcomes including physical, emotional, functional, and psychosocial data. These questionnaires were administered in paper format at the hospital. Overall, 38 patients participated in the survey and $66 \%$ of them completed it. Statistically significant symptom relief 
including improvement in patients' energy level, in the ability to perform daily tasks, and in the capacity to perform physical activities was observed at post-baseline assessments, including in the subgroup of patients undergoing ASCT after treatment with BV. The sustained improvement in HRQoL reported was indicative of good tolerability, however caution is required, because the method has not been validated and the sample size is small.

In the Phase III, AETHERA trial, HRQoL was evaluated using the European Quality of Life-5 Dimensions scale, with patients completing this questionnaire at several time points: at the beginning of each cycle, at the end of treatment, and every 3 months during follow-up. ${ }^{45}$ Results showed a modest negative impact of BV on HRQoL when compared to those reported by patients in the placebo arm, but differences did not reach statistical significance.

\section{Conclusion}

The introduction of BV to the treatment landscape of HL and ALCL has proven to be transformative and will allow expanding the therapeutic arsenal and improving the prognosis in these patients. Several aspects of BVassociated results are striking. First, it has a high level of single-agent antitumor activity. Second, the safety profile is outstanding, with PN being the most commonly reported AE, but it appears to be manageable with symptomatic treatment and dose modifications and did not result in an increase in discontinuation of study treatments or deaths. In fact, BV has been combined with currently used conventional chemotherapy regimens, and we already have data that BV can be safely added to several backbone regimens to increase efficacy and prolong durability of the response in these patients and provide them new therapeutic alternatives. ${ }^{46}$ Current trials are evaluating different combinations of BV with a variety of agents. Results of these studies may change the current standards of care for patients with HL. However, mature data needed for final conclusions are still pending.

The high response rate and favorable toxicity prolife of BV observed in the relapsed and refractory setting has allowed the rapid move of BV to the first-line therapy trials. In elderly patients with HL, BV alone or in combination may be a front-line option based on high response rate and favorable toxicity prolife. Likewise, preliminary data suggest that early consolidation with BV after ASCT improves PFS, so it seems to be a reasonable option in patients with HL who are at high risk of progression after SCT. In patients with HL, the administration of BV-based therapies in earlier phases of the disease could reduce the proportion of refractory $\mathrm{HL}$ and diminish the number of patients undergoing high-dose therapy and ASCT. In our opinion, this is a crucial point to reduce long-term toxicity of chemotherapy in these patients.

HL and ALCL patients had suffered years of scarce therapeutic progress. BV is the first monoclonal antibody approved for treating these diseases, ending a decades-long wait for a targeted approach. Amid all the exciting possibilities opened up with the incorporation of this new drug, we also need much caution to detect potential long-term effects of BV therapy, and as the drug becomes more prominent in the treatment of earlier stages of the disease, collection of such data ideally through randomized trials to shed light on this aspect will become increasingly important.

\section{Disclosure}

The authors report no conflicts of interest in this work.

\section{References}

1. Sieguel RL, Miller KD, Jemal A. Cancer statistics, 2017. CA Cancer J Clin. 2017;67(1):7-30.

2. Tilly H, Gaulard P, Lepage E, et al. Primary anaplastic large-cell lymphoma in adults: clinical presentation, immunophenotype, and outcome. Blood. 1997;90(9):3727-3734.

3. Stein H, Foss HD, Marafioti M, et al. CD30 anaplastic large cell lymphoma: a review of its histopathologic, genetic, and clinical features. Blood. 2000;96(12):3681-3695.

4. Senter PD, Sievers EL. The discovery and development of brentuximab vedotin for use in relapsed Hodgkin lymphoma and systemic anaplastic large cell lymphoma. Nat Biotechnol. 2012;30(7):631-637.

5. Yi JH, Kim SJ, Kim WS. Brentuximab vedotin: clinical updates and practical guidance. Blood Res. 2017;52(4):243-253.

6. Bartlett NL. Emerging role of novel therapies in Hodgkin lymphoma: proceed with caution. Hematology Am Soc Hematol Educ Program. 2017;2017(1):317-323.

7. Arai S, Fanale M, DeVos S, et al. Defining a Hodgkin lymphoma population for novel therapeutics after relapse from autologous hematopoietic cell transplant. Leuk Lymphoma. 2013;54(11):2531-2533.

8. Crump M. Management of Hodgkin lymphoma in relapse after autologous stem cell transplant. Hematology Am Soc Hematol Educ Program. 2008:326-333.

9. Younes A, Gopal AK, Smith SE, et al. Results of a pivotal phase II study of brentuximab vedotin for patients with relapsed or refractory Hodgkin's lymphoma. J Clin Oncol. 2012;30(18):2183-2189.

10. Chen R, Gopal AK, Smith SE, et al. Five-year survival and durability results of brentuximab vedotin in patients with relapsed or refractory Hodgkin lymphoma. Blood. 2016;128(12):1562-1566.

11. Moskowitz CH, Nademanee A, Masszi T, et al; AETHERA Study Group. Brentuximab vedotin as consolidation therapy after autologous stem-cell transplantation in patients with Hodgkin's lymphoma at risk of relapse or progression (AETHERA): a randomised, doubleblind, placebo-controlled, phase 3 trial. Lancet. 2015;385(9980): 1853-1862.

12. Sweetenham J, Walewski J, Nademanee AP, et al. Updated efficacy and safety data from the AETHERA trial of consolidation with brentuximab vedotin after autologous stem cell transplant (ASCT) in Hodgkin lymphoma patients at high risk of relapse. Blood. 2015; 126:3172. 
13. Chen R, Palmer JM, Martin P, et al. Results of a multicenter phase II trial of brentuximab vedotin as second-line therapy before autologous transplantation in relapsed/refractory Hodgkin lymphoma. Biol Blood Marrow Transplant. 2015;21(12):2136-2140.

14. Michallet AS, Guillermin Y, Deau B, et al. Sequential combination of gemcitabine, vinorelbine, pegylated liposomal doxorubicin and brentuximab as a bridge regimen to transplant in relapsed or refractory Hodgkin lymphoma. Haematologica. 2015;100(7):e269-e271.

15. Moskowitz AJ, Schoder H, Yahalom J, et al. PET-adapted sequential salvage therapy with brentuximab vedotin followed by augmented ifosamide, carboplatin, and etoposide for patients with relapsed and refractory Hodgkin's lymphoma: a non-randomised, open-label, singlecentre, phase 2 study. Lancet Oncol. 2015;16(3):284-292.

16. Cassaday RD, Fromm J, Cowan AJ, et al. Safety and activity of brentuximab vedotin plus ifosfamide, carboplatin, and etposide (ICE) for relapsed/refractory classical Hodgkin lymphoma: initial results of a phase I/II trial. Blood. 2016;128:1834.

17. LaCasce AS, Bociek G, Sawas A, et al. Brentuximab vedotin plus bendamustine: a highly active salvage treatment regimen for patients with relapsed or refractory Hodgkin lymphoma. Blood. 2015;126:3982.

18. Garcia-Sanz R, Sureda A, Gonzalez AP, et al. Brentuximab vedotin plus ESHAP (BESHAP) is a highly effective combination for inducing remission in refractory and relapsed Hodgkin lymphoma patients prior to autologous stem cell transplant: a trial of the Spanish Group of Lymphoma and Bone Marrow Transplantation (GELTAMO). Blood. 2016; 128:1109.

19. Herrera AF, Bartlett NL, Ramchandren R, et al. Preliminary results from a phase $1 / 2$ study of brentuximab vedotin in combination with nivolumab in patients with relapsed or refractory Hodgkin lymphoma. Blood. 2016;128:1105.

20. Diefenbach CS, Hong F, David KA, et al. A phase I study with an expansion cohort of the combination of Ipilimumab an nivolumab and brentuximab vedotin in patients with relapse/refractory Hodgkin lymphoma: a trial of the ECOG-ACRIN Cancer Research Group (E4412 Arms D and E). Blood. 2016;128:1106.

21. Bartlett NL, Chen R, Fanale MA, et al. Retretament with brentuximab vedotin in patients with CD30-positive hematologic malignancies. J Hematol Oncol. 2014;7:1-8.

22. Forero-Torres A, Holkova B, Goldschmidt J, et al. Phase 2 study of frontline brentuximab vedotin monotherapy in Hodgkin lymphoma patients aged 60 years and older. Blood. 2015;126(26):2798-2804.

23. Gibb A, Pirrie S, Linton K, et al. Results of phase II study of brentuximab vedotin in the first line treatment of Hodgkin lymphoma patients considered unsuitable for standard chemotherapy (BREVITY). Hematol Oncol. 2017;35(Suppl 2):80-81.

24. Gallamini A, Bijou F, Viotti J, et al. Brentuximab-vedotin and bendamustine is a feasible and effective drugs combination as first-line treatment of Hodgkin lymphoma in the elderly (HALO TRIAL). Hematol Oncol. 2017;35(Suppl 2):170.

25. Friedberg JW, Forero-Torres A, Bordoni RE, et al. Frontline brentuximab vedotin in combination with dacarbazine or bendamustine in patients aged $\geq 60$ years with HL. Blood. 2017;130(26):2829-2837.

26. Younes A, Connors JM, Park SI, et al. Brentuximab vedotin combined with ABVD or AVD for patients with newly diagnosed Hodgkin's lymphoma: a phase 1, open-label, dose-escalation study. Lancet Oncol. 2013; 14(13):1348-1356.

27. Connors JM, Ansell S, Fanale MA, Park SI, Younes A. Five-year follow-up of brentuximab vedotin combined with ABVD or AVD for advanced-stage classical Hodgkin lymphoma. Blood. 2017;130(11): 1375-1377.

28. Kumar A, Casulo C, Yahalom J, et al. Brentuximab vedotin and AVD followed by involved-site radiotherapy in early stage, unfavorable risk Hodgkin lymphoma. Blood. 2016;128(11):1458-1464.
29. Connors JM, Jurczak W, Straus DJ, et al. Brentuximab vedotin with chemotherapy for stage III or IV hodgkin's lymphoma. $N$ Engl J Med. 2018;378(4):331-344.

30. Borchmann P, Eichenauer DA, Pluetschow A, et al. Targeted BEACOPP variants in patients in patients with newly diagnosed advanced stage classical Hodgkin lymphoma: final analysis of a randomized phase II study. Blood. 2015;126:580.

31. Tsuyama N, Sakamoto K, Sakata S, Dobashi A, Takeuchi K. Anaplastic large cell lymphoma: pathology, genetics, and clinical aspects. J Clin Exp Hematop. 2017;57(3):120-142.

32. Savage KJ, Harris NL, Vose JM, et al. ALK anaplastic large-cell lymphoma is clinically and immunophenotypically different from both ALK+ ALCL and peripheral T-cell lymphoma, not otherwise specified: report from the International Peripheral T-Cell Lymphoma Project. Blood. 2008;111(12):5496-5504.

33. Smith SM, Burns LJ, van Besien K, et al. Hematopoietic cell transplantation for systemic mature T-cell non-Hodgkin lymphoma. J Clin Oncol. 2013;31(25):3100-3109.

34. Morel A, Brière J, Lamant L, et al. Long-term outcomes of adults with first-relapsed/refractory systemic anaplastic large-cell lymphoma in the pre-brentuximab vedotin era: a LYSA/SFGM-TC study. Eur J Cancer. 2017;83:146-153.

35. Younes A, Bartlett NL, Leonard JP, et al. Brentuximab vedotin (SGN-35) for relapsed CD30-positive lymphomas. N Engl J Med. 2010; 363(19):1812-1821.

36. Pro B, Advani R, Brice P, et al. Brentuximab vedotin (SGN-35) in patients with relapsed or refractory systemic anaplastic large-cell lymphoma: results of a phase II study. J Clin Oncol. 2012;30(18):2190-2196.

37. Pro B, Advani R, Brice P. Five-year results of brentuximab vedotin in patients with relapsed or refractory systemic anaplastic large cell lymphoma. Blood. 2017;130(25):2709-2717.

38. Broccoli A, Pellegrini C, Di Rocco A, et al. Italian real-life experience with brentuximab vedotin: results of a large observational study of 40 cases of relapsed/refractory systemic anaplastic large cell lymphoma. Haematologica. 2017;102(11):1931-1935.

39. Ferreri AJ, Govi S, Pileri SA, Savage KJ. Anaplastic large cell lymphoma ALK-negative. Crit Rev Oncol Hematol. 2013;85(2):206-215.

40. Chen X, Soma LA, Fromm JR. Targeted therapy for Hodgkin lymphoma and systemic anaplastic large cell lymphoma: focus on brentuximab vedotin. Onco Targets Ther. 2013;7:45-56.

41. Fanale MA, Horwitz SM, Forero-Torres A, et al. Five-year outcomes for frontline brentuximab vedotin with CHP for CD30-expressing peripheral T-cell lymphomas. Blood. 2018;131(9):2120-2124.

42. Zinzani PL, Sasse S, Radford J, Shonukan O, Bonthapally V. Experience of brentuximab vedotin in relapsed/refractory Hodgkin lymphoma and relapsed/refractory systemic anaplastic large-cell lymphoma in the Named Patient Program: review of the literature. Crit Rev Oncol Hematol. 2015;95(3):359-369.

43. Perrot A, Monjanel H, Bouabdallah R, et al; Lymphoma Study Association (LYSA). Impact of post-brentuximab vedotin consolidation on relapsed/refractory CD30+ Hodgkin lymphomas: a large retrospective study on 240 patients enrolled in the French Named-Patient Program. Haematologica. 2016;101(4):466-473.

44. Chen R, Allibone S, Bartlett NL, et al. Patient-reported outcomes of brentuximab vedotin in Hodgkin lymphoma and anaplastic large-cell lymphoma. Onco Targets Ther. 2016;9:2027-2034.

45. Ramsey SD, Nademanee A, Masszi T, et al. Quality of life results from a phase 3 study of brentuximab vedotin consolidation following autologous haematopoietic stem cell transplant for persons with Hodgkin lymphoma. Br J Haematol. 2016;175(5):860-867.

46. Donato EM, Fernández-Zarzoso M, De La Rubia J. Immunotherapy for the treatment of Hodgkin lymphoma. Expert Rev Hematol. 2017; 10(5):417-423. 


\section{Publish your work in this journal}

OncoTargets and Therapy is an international, peer-reviewed, open access journal focusing on the pathological basis of all cancers, potential targets for therapy and treatment protocols employed to improve the management of cancer patients. The journal also focuses on the impact of management programs and new therapeutic agents and protocols on

patient perspectives such as quality of life, adherence and satisfaction. The manuscript management system is completely online and includes a very quick and fair peer-review system, which is all easy to use. Visit http://www.dovepress.com/testimonials.php to read real quotes from published authors.

Submit your manuscript here: http://www.dovepress.com/oncotargets-and-therapy-journal 